\title{
Inflation with a running spectral index in supergravity
}

\author{
M. Kawasaki \\ Research Center for the Early Universe, \\ University of Tokyo, Tokyo, 113-0033, Japan \\ Masahide Yamaguchi \\ Physics Department, Brown University, Providence, Rhode Island 02912 \\ Jun'ichi Yokoyama \\ Department of Earth and Space Science, Graduate School of Science, \\ Osaka University, Toyonaka 560-0043, Japan
}

(Dated: May 30, 2018)

\begin{abstract}
The first year Wilkinson Microwave Anisotropy Probe data favors primordial adiabatic fluctuation with a running spectral index with $n_{s}>1$ on a large scale and $n_{s}<1$ on a smaller scale. The model building of inflation that predicts perturbations with such a spectrum is a challenge, because most models predict fluctuations with either $n_{s}>1$ (hybrid inflation) or $n_{s}<1$ (new, chaotic, or topological inflation). We give a sensible particle physics model in supergravity that accommodates the desired running of the spectral index using double inflation.
\end{abstract}

PACS numbers: 98.80.Cq,04.65.+e,11.27.+d

RESCEU-10/03, BROWN-HET-1352, OU-TAP-202 


\section{INTRODUCTION}

The first year data release of the Wilkinson Microwave Anisotropy Probe (WMAP) has opened a new era of high-precision cosmology [1]. It has not only confirmed the "concordance" values of the cosmological parameters with much smaller uncertainties than before but also extracted important information on the primordial spectrum of density perturbations [2]. It has been reported that [3] their result favors purely adiabatic fluctuations with a remarkable feature that the spectral index runs from $n_{s}>1$ on a large scale to $n_{s}<1$ on a smaller scale. More specifically they obtain $n_{s}=1.10_{-0.06}^{+0.07}$ and $d n_{s} / d \ln k=-0.042_{-0.020}^{+0.021}$ on the scale $k_{0}=0.002 \mathrm{Mpc}^{-1}$.

It is not straightforward to make a model of inflation [4] that predicts perturbations with such a spectrum. In a single-field slow-roll inflation model with a potential $V[\phi]$, the amplitude of curvature perturbation in the comoving gauge $\mathcal{R}$ [5] generated on the comoving scale $r=2 \pi / k$ is given by

$$
\mathcal{R}(k)=\frac{1}{2 \pi} \frac{H^{2}\left(t_{k}\right)}{\left|\dot{\phi}\left(t_{k}\right)\right|}, \quad H^{2}\left(t_{k}\right)=\frac{V\left[\phi\left(t_{k}\right)\right]}{3 M_{G}^{2}},
$$

where $t_{k}$ is the epoch $k$ mode left the Hubble radius during inflation [6]. The spectral index is defined and given by

$$
n_{s}-1 \equiv \frac{d \ln |\mathcal{R}(k)|^{2}}{d \ln k} \cong-6 \epsilon+2 \eta, \quad \epsilon \equiv \frac{M_{G}^{2}}{2}\left(\frac{V^{\prime}[\phi]}{V[\phi]}\right)^{2}, \quad \eta \equiv \frac{M_{G}^{2} V^{\prime \prime}[\phi]}{V[\phi]}
$$

We also find

$$
\frac{d n_{s}}{d \ln k}=16 \epsilon \eta-24 \epsilon^{2}-2 \xi, \quad \xi \equiv M_{G}^{4} \frac{V^{\prime \prime \prime}[\phi] V^{\prime}[\phi]}{V[\phi]^{2}} .
$$

From Eqs. (2) we find that $\eta$ or the effective mass of the inflaton $\phi$ must change significantly to achieve running of the spectral index from $n_{s}>1$ to $n_{s}<1 .{ }^{1}$ Here $M_{G}=2.4 \times 10^{18} \mathrm{GeV}$ is the reduced Planck mass. Hereafter we take $M_{G}=1$.

One possibility to realize such a spectral feature is a hybrid inflation model of Linde and Riotto, in which supergravity effect becomes dominant in the early stage of inflation and one-loop effect plays an important role in the final stage of inflation [7]. Unfortunately, however, although the spectral index crosses unity in this model, its variation is too mild to reproduce the WMAP result for a sensible set of model parameters. ${ }^{2}$ This is mainly because the Yukawa coupling constant must be relatively small for sufficient inflation as we argue below.

In order to circumvent this problem we consider a double inflation model combining hybrid inflation [10] and new inflation [11] in supergravity. In our model the first stage of hybrid inflation proceeds in the same way as the Linde-Riotto model [7], which is a supergravity version of a supersymmetric model [12, 13], and the desired spectrum with a running index from $n_{s}>1$ to $n_{s}<1$ is generated then. At the same time the initial

\footnotetext{
${ }^{1}$ Recently, Feng et al. discussed an inflation model in the context of an extra dimension and showed that a large variation of spectral index can be realized [8].

2 Recently, Kyae and Shafi discussed hybrid inflation in four- and five- dimensional grand unified theories. But it is shown that the running of the spectral index is too mild also in this model [9].
} 
condition for subsequent new inflation is naturally prepared [14], which continues about 40 $e$-folds to expand the comoving scale with the desired spectral shape of density fluctuation to larger scales observable with WMAP. Furthermore, new inflation predicts sufficiently a low reheating temperature to avoid overproduction of gravitinos.

The rest of the paper is organized as follows. In Sec. II we review the supergravity hybrid inflation model of Linde and Riotto [7] and argue its difficulty to realize a significantly large running of the spectral index on scales probed by WMAP. Then in Sec. III we introduce our double inflation model which was originally proposed in [14] and utilized to realize the formation of primordial black holes [15] or to fit observations of large-scale structure [16]. Section IV is devoted to a discussion and future outlook.

\section{HYBRID INFLATION IN SUPERGRAVITY}

Here we first review the supergravity hybrid inflation model of Linde and Riotto 7]. The superpotential is given by

$$
W_{H}=\lambda S \bar{\Psi} \Psi-\mu^{2} S
$$

where $S$ is a gauge-singlet superfield, while $\Psi$ and $\bar{\Psi}$ are a conjugate pair of superfields transforming as nontrivial representations of some gauge group, and $\lambda$ and $\mu$ are positive parameters much smaller than unity. This system respects the $R$ symmetry under which they are transformed as $S \longrightarrow e^{2 i \alpha} S, \Psi \longrightarrow e^{2 i \alpha} \Psi, \bar{\Psi} \longrightarrow e^{-2 i \alpha} \bar{\Psi}$, and $W_{H} \longrightarrow e^{2 i \alpha} W_{H}$. The $R$-invariant Kähler potential is given by

$$
K_{H}=|S|^{2}+|\Psi|^{2}+|\bar{\Psi}|^{2}+\cdots
$$

Here, for simplicity, we have neglected higher-order terms, which make the calculation complicated but do not change the essential result.

The potential of scalar components of the superfields $z_{i}$ in supergravity is given by

$$
V=e^{K}\left\{\left(\frac{\partial^{2} K}{\partial z_{i} \partial z_{j}^{*}}\right)^{-1} D_{z_{i}} W D_{z_{j}^{*}} W^{*}-3|W|^{2}\right\}+V_{D},
$$

with

$$
D_{z_{i}} W=\frac{\partial W}{\partial z_{i}}+\frac{\partial K}{\partial z_{i}} W
$$

Here the scalar components of the superfields are denoted by the same symbols as the corresponding superfields, and $V_{D}$ represents the $D$-term contribution. From Eqs. (4) and (5) we explicitly find

$$
\begin{aligned}
V_{H}(S, \Psi, \bar{\Psi})= & e^{|S|^{2}+|\Psi|^{2}+|\bar{\Psi}|^{2}}\left\{\left(1-|S|^{2}+|S|^{4}\right)\left|\lambda \bar{\Psi} \Psi-\mu^{2}\right|^{2}\right. \\
& \left.+|S|^{2}\left[\left|\lambda\left(1+|\Psi|^{2}\right) \bar{\Psi}-\mu^{2} \Psi^{*}\right|^{2}+\left|\lambda\left(1+|\bar{\Psi}|^{2}\right) \Psi-\mu^{2} \bar{\Psi}^{*}\right|^{2}\right]\right\}+V_{D H}
\end{aligned}
$$

Since the above potential does not depend on the phase of the complex scalar field $S$, we identify its real part, $\sigma \equiv \operatorname{Re} S / \sqrt{2}$, with the inflaton. Composing the mass matrix of $\Psi$ and $\bar{\Psi}$ from Eq. (8), we find that the eigenvalues and the corresponding eigenstates are given by

$$
M_{ \pm}^{2}=\lambda^{2}|S|^{2} \pm \lambda \mu^{2}=\frac{\lambda^{2}}{2} \sigma^{2} \pm \lambda \mu^{2}, \quad \text { for } \quad \Psi^{*}=\mp \bar{\Psi}
$$


where we have assumed $\lambda \gg \mu$. Since these eigenstates are along $D$-flat directions, the scalar fields will eventually start to roll, keeping $\Psi^{*}=\bar{\Psi}$, as $\sigma$ becomes smaller than the critical value $\sigma_{c} \equiv \sqrt{2} \mu / \sqrt{\lambda}$.

Then under the condition $\Psi^{*}=\bar{\Psi}$, the potential reduces to a familiar form of the typical hybrid inflation potential:

$$
V_{H}=\left(\lambda|\Psi|^{2}-\mu^{2}\right)^{2}+\lambda^{2} \sigma^{2}|\Psi|^{2}+\frac{1}{8} \mu^{4} \sigma^{4}+\cdots
$$

For $\sigma>\sigma_{c}$, the potential is minimized at $\Psi=\bar{\Psi}=0$ and inflation is driven by the false vacuum energy density $\mu^{4}$.

As a result of the mass split of scalar multiplets composed by $\Psi$ and $\bar{\Psi}$ with mass squared $M_{ \pm}^{2}$ and their superpartner fermions with mass $M=\lambda \sigma / \sqrt{2}$, the radiative correction to the potential is non-negligible during inflation. The one-loop correction reads [13]

$$
V_{1 L}=\frac{\lambda^{2}}{128 \pi^{2}}\left[\left(\lambda \sigma^{2}+2 \mu^{2}\right)^{2} \ln \frac{\lambda \sigma^{2}+2 \mu^{2}}{\Lambda^{2}}+\left(\lambda \sigma^{2}-2 \mu^{2}\right)^{2} \ln \frac{\lambda \sigma^{2}-2 \mu^{2}}{\Lambda^{2}}-2 \lambda^{2} \sigma^{4} \ln \frac{\lambda \sigma^{2}}{\Lambda^{2}}\right]
$$

where $\Lambda$ is the renormalization scale. When $\sigma \gg \sigma_{c}$ it is approximated as

$$
V_{1 L} \cong \frac{\lambda^{2} \mu^{4}}{8 \pi^{2}} \ln \frac{\sigma}{\sigma_{c}}
$$

As a result the effective potential of the inflaton $\sigma$ during hybrid inflation reads

$$
V_{H}[\sigma] \cong \mu^{4}\left(1+\frac{\lambda^{2}}{8 \pi^{2}} \ln \frac{\sigma}{\sigma_{c}}+\frac{1}{8} \sigma^{4}+\cdots\right)
$$

As long as the field amplitude is sub-Planck scale or smaller than unity, the effective potential is dominated by the false vacuum energy. Comparing the derivative of the second term of the right-hand side and that of the last term, we find that the dynamics of the scalar field is dominated by the nonrenormalizable term for $\sigma>\sqrt{\lambda /(2 \pi)} \equiv \sigma_{d}$ and by the radiative correction for $\sigma<\sigma_{d}$. Since we are assuming $\mu \ll \lambda \ll 1$, we find $\sigma_{c} \ll \sigma_{d} \ll 1$. In this situation, the number of $e$-folds of exponential expansion during inflation, $N_{H}$, is sensitive only to $\sigma_{d}$ and given by

$$
N_{H}=\int_{\sigma_{c}}^{\sigma_{i}} \frac{V_{H}[\sigma]}{V_{H}^{\prime}[\sigma]} d \sigma \cong \int_{\sigma_{d}}^{\sigma_{i}} \frac{2}{\sigma^{3}} d \sigma+\int_{\sigma_{c}}^{\sigma_{d}} \frac{8 \pi^{2}}{\lambda^{2}} \sigma d \sigma \cong \frac{4 \pi}{\lambda}
$$

where $\sigma_{i}$ is the initial value at the onset of hybrid inflation. Although the approximate expression for the one-loop potential (12) is valid only for $\sigma \gg \sigma_{c}$, the fact that the resultant expression of $N_{H}$ is independent of $\sigma_{c}$ implies that the above formula (14) gives a reasonable approximation for the actual number of $e$-folds. We also note that the amount of inflation during $\sigma>\sigma_{d}$ and that during $\sigma<\sigma_{d}$ are about the same with the $e$-folds $\approx 2 \pi / \lambda$. Thus in order to achieve sufficiently long inflation to solve the horizon and the flatness problems, $N_{H} \gtrsim 60, \lambda$ should be rather small: $\lambda \lesssim 4 \pi / 60 \simeq 0.2$.

Calculating the slow-roll parameters $\epsilon, \eta$, and $\xi$ for the potential (13), we find

$$
\epsilon=\mathcal{O}\left(\left(\frac{\sigma}{M_{G}}\right)^{6}\right), \quad \eta=\mathcal{O}\left(\left(\frac{\sigma}{M_{G}}\right)^{2}\right), \quad \xi=\mathcal{O}\left(\left(\frac{\sigma}{M_{G}}\right)^{4}\right)
$$


for $\sigma \sim \sigma_{d}$ in dimensionful units, so that the spectral index of scalar perturbation and its variation are given by

$$
\begin{aligned}
n_{s}-1 & =-6 \epsilon+2 \eta \cong 2 \eta=3 \sigma^{2}-\frac{\sigma_{d}^{4}}{\sigma^{2}}, \\
\frac{d n_{s}}{d \ln k} & =16 \epsilon \eta-24 \epsilon^{2}-2 \xi \cong-2 \xi=-\left(\frac{\sigma_{d}^{8}}{\sigma^{4}}+4 \sigma_{d}^{4}+3 \sigma^{4}\right) .
\end{aligned}
$$

As is seen here, this model has the desired feature of the running spectral index toward smaller values for decreasing length scales qualitatively. The spectral index crosses unity at $\sigma=\sigma_{d} / 3^{1 / 4} \sim 0.8 \sigma_{d}$. In order that this happens on the observable scales by WMAP and large-scale structures, one should have about $50 \mathrm{e}$-folds of inflation after $\sigma$ crossed this value, which requires $\lambda \sim 0.1$ from Eq. (14). As a result, we find that the amplitude of spectral running is too small:

$$
\frac{d n_{s}}{d \ln k} \sim-10^{-3}
$$

Conversely, this quantity could be larger and match observation if we adopted a larger value of the coupling constant $\lambda$. Indeed from Eqs. (16) and (17) we find

$$
\begin{aligned}
\sigma_{d} & =\frac{1}{2 \sqrt{2}}\left\{\left[\frac{4}{9}\left(n_{s}-1\right)^{4}-\frac{32}{3}\left(n_{s}-1\right)^{2} \frac{d n_{s}}{d \ln k}\right]^{\frac{1}{2}}-\frac{14}{3}\left(n_{s}-1\right)^{2}-8 \frac{d n_{s}}{d \ln k}\right\}^{\frac{1}{4}}, \\
\sigma & =\left[\frac{n_{s}-1}{2}+\frac{1}{2\left(n_{s}-1\right)} \frac{d n_{s}}{d \ln k}+\frac{4 \sigma_{d}^{4}}{n_{s}-1}\right]^{\frac{1}{2}},
\end{aligned}
$$

where $\sigma$ is the field amplitude when the relevant scale left the Hubble radius during hybrid inflation. Using the central values obtained by WMAP on a comoving scale, $k_{0}=0.002 \mathrm{Mpc}^{-1}$, $n_{s}-1=0.1$ and $d n_{s} / d \ln k=-0.042$, we find $\sigma_{d}=0.27$ or $\lambda=0.47$ and $\sigma=0.25=0.91 \sigma_{d}$. This means that the $e$-folds of hybrid inflation after the comoving scale with the observed spectral shape has crossed the Hubble radius is only about 11.3. Hence we must invoke another inflation to push the relevant scale to the scale $k_{0}=0.002 \mathrm{Mpc}^{-1}$. If this is achieved, we can find the energy scale of hybrid inflation from the amplitude of curvature fluctuation at $\sigma=0.25$,

$$
\mathcal{R}\left(k_{0}\right)=\frac{\mu^{2}}{\sqrt{3} \pi}\left(\frac{\sigma_{d}^{4}}{\sigma}+\sigma^{3}\right)^{-1}=4.9 \mu^{2}=4.8 \times 10^{-5},
$$

corresponding to $A=0.77$ of $[3]$. We therefore find $\mu=3.1 \times 10^{-3}=7.5 \times 10^{15} \mathrm{GeV}$.

Although we have taken the central values obtained by WMAP, the $n_{s}-1$ and $d n_{s} / d \ln k$ are determined with rather large uncertainties. For example, if we take $d n_{s} / d \ln k=-0.021$ corresponding to $68 \%$ probability lower bound, we obtain $\sigma_{d}=0.22\left(\lambda=0.30, \sigma=0.95 \sigma_{d}\right)$, which changes the $e$-folds of the hybrid inflation from 11.3 to 19.9. Thus, we should take $\Delta N_{H} \simeq 8$ as the uncertainty of $e$-folds of hybrid inflation.

Note also that the two contributions from the supergravity effect and the one-loop correction are dealt with separately in the above analytic estimate. However, of course, both contributions should be considered simultaneously. So we have calculated the fluctuations numerically. Then, we have found that the best-fit parameters to reproduce the WMAP results are slightly changed and given by $\mu=2.9 \times 10^{-3}=7.2 \times 10^{15} \mathrm{GeV}, \lambda=0.43$. The results are shown in Figs. 1].3. In this case, the $e$-folds of hybrid inflation after the comoving scale with the observed spectral shape has crossed the Hubble radius is only about 10.3. 


\section{HYBRID NEW INFLATION}

In order to push the scales with the desired spectral shape of density fluctuations to cosmologically observable scales, we consider new inflation which occurs after hybrid inflation discussed above. One should notice that, generally speaking, hybrid inflation predicts a high reheating temperature because the inflaton has gauge couplings and its energy scale is relatively high usually. On the other hand, new inflation predicts a sufficiently low reheating temperature to avoid overproduction of gravitinos. Thus, the occurrence of new inflation following hybrid inflation is favorable also in this respect. Furthermore, in our model, the initial value of new inflation is dynamically set during hybrid inflation, which evades the severe initial value problem of new inflation. In this section, we first review the superpotential and Kähler potential for the new inflation sector and its dynamics [14], and then combine these two inflation by considering full superpotential.

We introduce a chiral superfield $\Phi$ with an $R$ charge $2 /(n+1)$, but assume that the $\mathrm{U}(1)_{R}$ symmetry is dynamically broken to a discrete $Z_{2 n} R$ symmetry at a scale $v \ll 1$. The superpotential of this sector therefore reads

$$
W_{N}[\Phi]=v^{2} \Phi-\frac{g}{n+1} \Phi^{n+1}
$$

with $g$ being a coupling constant of order of unity. We assume that both $g$ and $v$ are real and positive for simplicity. The $R$-invariant Kähler potential is given by

$$
K_{N}=|\Phi|^{2}+\frac{c_{N}}{4}|\Phi|^{4}+\cdots
$$

where $c_{N}$ is a constant smaller than unity. We assume that there are no direct interactions between fields relevant to hybrid inflation discussed in the previous section and $\Phi$. Hence the full superpotential and Kähler potential read $W=W_{H}+W_{N}$ and $K=K_{H}+K_{N}$, respectively.

Before performing the full analysis we review how new inflation proceeds in this model. The scalar potential given from Eqs. (22) and (23) reads

$$
\begin{aligned}
V_{N}[\Phi]= & \frac{\exp \left(|\Phi|^{2}+\frac{c_{N}}{4}|\Phi|^{4}\right)}{1+c_{N}|\Phi|^{2}} \\
\times & {\left[\left|\left(1+|\Phi|^{2}+\frac{c_{N}}{2}|\Phi|^{4}\right) v^{4}-\left(1+\frac{|\Phi|^{2}}{n+1}+\frac{c_{N}|\Phi|^{4}}{2(n+1)}\right) g \Phi^{n}\right|^{2}\right.} \\
& \left.-3\left(1+c_{N}|\Phi|^{2}\right)|\Phi|^{2}\left|v^{2}-\frac{g}{n+1} \Phi^{n}\right|^{2}\right]
\end{aligned}
$$

It has a minimum at

$$
|\Phi|_{\min } \cong\left(\frac{v^{2}}{g}\right)^{\frac{1}{n}} \quad \text { and } \operatorname{Im} \Phi_{\min }^{n}=0
$$

with a negative energy density

$$
V_{N}\left[\Phi_{\min }\right] \cong-3 e^{K_{N}}\left|W_{N}\left[\Phi_{\min }\right]\right|^{2} \cong-3\left(\frac{n}{n+1}\right)^{2} v^{4}\left|\Phi_{\min }\right|^{2}
$$


Assuming that this negative value is canceled by a positive contribution due to supersymmetry breaking, $\Lambda_{\text {SUSY }}^{4}$, we can relate energy scale of this model with the gravitino mass $m_{3 / 2}$ as

$$
m_{3 / 2} \cong \frac{n}{n+1}\left(\frac{v^{2}}{g}\right)^{\frac{1}{n}} v^{2}
$$

Without loss of generality we may identify the real part of $\Phi$ with the inflaton $\phi \equiv$ $\operatorname{Re} \Phi / \sqrt{2}$. The dynamics of inflaton is governed by the lower-order potential

$$
V_{N}[\phi] \cong v^{4}-\frac{c_{N}}{2} v^{4} \phi^{2}-\frac{2 g}{2^{n / 2}} v^{2} \phi^{n}+\frac{g^{2}}{2^{n}} \phi^{2 n} .
$$

Since the last term is negligible during inflation and the Hubble parameter is dominated by the first term, $H=v^{2} / \sqrt{3}$, the slow-roll equation of motion reads

$$
3 H \dot{\phi}=-V_{N}^{\prime}[\phi] \cong-c_{N} v^{4} \phi-2^{\frac{2-n}{2}} n g v^{2} \phi^{n-1},
$$

and the slow-roll parameters are given by

$$
\epsilon \cong \frac{1}{2}\left(c_{N} \phi+2^{\frac{2-n}{2}} n g \frac{\phi^{n-1}}{v^{2}}\right)^{2}, \quad \eta=-c_{N}-2^{\frac{2-n}{2}} n(n-1) g \frac{\phi^{n-2}}{v^{2}},
$$

in this new inflation regime. Thus inflation is realized with $c_{N} \ll 1$ and ends at

$$
\phi=\sqrt{2}\left(\frac{\left(1-c_{N}\right) v^{2}}{g n(n-1)}\right)^{\frac{1}{n-2}} \equiv \phi_{e}
$$

when $|\eta|$ becomes as large as unity.

Since the two terms on the right-hand side of Eq. (29) are identical at

$$
\phi=\sqrt{2}\left(\frac{c_{N} v^{2}}{g n}\right)^{\frac{1}{n-2}} \equiv \phi_{d}
$$

the number of $e$-folds of new inflation is estimated as

$$
N_{N}=-\int_{\phi_{i}}^{\phi_{e}} \frac{V_{N}[\phi]}{V_{N}^{\prime}[\phi]} d \phi \cong \int_{\phi_{i}}^{\phi_{d}} \frac{d \phi}{c_{N} \phi}+\int_{\phi_{d}}^{\phi_{e}} \frac{2^{\frac{n-2}{2}} v^{2}}{g n \phi^{n-1}} d \phi=\frac{1}{c_{N}} \ln \frac{\phi_{d}}{\phi_{i}}+\frac{1-n c_{N}}{(n-2) c_{N}\left(1-c_{N}\right)}
$$

for $0<c_{N}<n^{-1}$. If $c_{N}$ vanishes, we instead find

$$
N_{N}=\int_{\phi_{i}}^{\phi_{e}} \frac{2^{\frac{n-2}{2}} v^{2}}{g n \phi^{n-1}} d \phi=\frac{2^{\frac{n-2}{2}} v^{2}}{g n(n-2)} \phi_{i}^{2-n}-\frac{n-1}{n-2} .
$$

Here $\phi_{i}$ is the initial value of $\phi$, whose determination mechanism we now argue.

In the hybrid inflation stage $\sigma>\sigma_{c}$, the cosmic energy density is dominated by the false vacuum energy $\mu^{4}$ with $\Psi=\bar{\Psi}=0$. Hence, in the full scalar potential, which is obtained by the prescription (6) with $K=K_{H}+K_{N}$ and $W=W_{H}+W_{N}$, the interaction terms between $\Phi$ and the hybrid inflation sector are given by

$$
V \supset \mu^{4}|\Phi|^{2}+\mu^{2} v^{2}\left(\Phi^{*} S+\Phi S^{*}\right)+\cdots=\frac{1}{2} \mu^{4}\left(\phi^{2}+\chi^{2}\right)+\mu^{2} v^{2} \sigma \phi+\cdots,
$$


where $\phi=\operatorname{Re} \Phi / \sqrt{2}$ and $\chi=\operatorname{Im} \Phi / \sqrt{2}$. Hence at the end of hybrid inflation, $\sigma=\sigma_{c}, \phi$ and $\chi$ have a minimum at

$$
\phi_{\min } \cong-\frac{v^{2}}{\mu^{2}} \sigma_{c}=-\sqrt{\frac{2}{\lambda}} \frac{v^{2}}{\mu} \text { and } \quad \chi_{\min } \cong 0,
$$

respectively. Since the effective mass is larger than the Hubble parameter during hybrid inflation, the above configuration is realized with the dispersion

$$
\left\langle\left(\phi-\phi_{\min }\right)^{2}\right\rangle=\left\langle\chi^{2}\right\rangle=\frac{3}{8 \pi^{2}} \frac{H_{H}^{4}}{\mu^{4}}=\frac{\mu^{4}}{24 \pi^{2}},
$$

due to quantum fluctuations [17]. Here $\phi$ will eventually relax to this minimum during hybrid inflation. The ratio of quantum fluctuation to the expectation value should satisfy

$$
\frac{\sqrt{\left\langle\left(\phi-\phi_{\min }\right)^{2}\right\rangle}}{\left|\phi_{\min }\right|}=\frac{\sqrt{\lambda}}{4 \sqrt{3} \pi}\left(\frac{\mu}{v}\right)^{2} \mu \ll 1,
$$

so that the initial value of the inflaton for new inflation is located off the origin with an appropriate magnitude.

When $\sigma$ reaches $\sigma_{c}$, a phase transition takes place and hybrid inflation ends immediately, which is followed by field oscillation of $S$ and $\Psi(\bar{\Psi})$ and their decay. If this oscillation phase lasts for a prolonged period due to gravitationally suppressed interactions of these fields, $\phi$ will also oscillate and its amplitude decreases with an extra factor $v / \mu$ 15]. In this case, new inflation could start with a even smaller value of $\phi$ depending on its phase of oscillation at the onset of inflation (see [16] for an analytic estimate of the initial phase). So we set the initial value of $\phi$ as

$$
\phi_{i}=\sqrt{\frac{2}{\lambda}} \frac{v^{3}}{\mu^{2}}
$$

and new inflation occurs until $\phi=\phi_{e}$ with the potential (28). Thus we can understand the evolution of the universe throughout double inflation.

Contrary to the hybrid inflation regime we do not have much precise observational constraints on the new inflation regime, so we cannot fully specify values of the model parameters for new inflation. Hence let us content ourselves with a few specific examples. First we consider the cases with $c_{N}=0$. Then from Eq. (34) the number of $e$-folds of new inflation reads

$$
N_{N}=\frac{\lambda^{\frac{n-2}{2}} \mu^{2 n-4} v^{8-3 n}}{n(n-2) g}-\frac{n-1}{n-2} \cong \frac{\lambda^{\frac{n-2}{2}} \mu^{2 n-4} v^{8-3 n}}{n(n-2) g} .
$$

This should be around $40+(2 / 3) \ln (\mu / v)$ to push the comoving scale with appropriate spectral shape to the appropriate physical length scale. ${ }^{3}$ On the other hand, the amplitude of curvature perturbation at the onset of new inflation, $\phi=\phi_{\min }$, is given by

$$
\mathcal{R}=\frac{\lambda^{\frac{n-1}{2}} \mu^{2 n-2} v^{7-3 n}}{2 \sqrt{6} \pi n g}=\frac{n-2}{2 \sqrt{6} \pi} \sqrt{\lambda} \mu \frac{\mu}{v} N_{N} \cong 4.9 \times 10^{-3}(n-2) \frac{\mu}{v} \frac{N_{N}}{40},
$$

\footnotetext{
3 Comoving scales that left the Hubble radius in the late stage of hybrid inflation reenter the horizon before the beginning of the new inflation. Hence extra $e$-folds $(2 / 3) \ln (\mu / v)$ should be added in making a correspondence between comoving horizon scales during hybrid inflation and proper scales [15].
} 
where use has been made of the values $\lambda=0.43$ and $\mu=2.9 \times 10^{-3}$ in the last equality. From Eq. (40) we find

$$
\begin{aligned}
& v=5.5 \times 10^{-4}\left(\frac{g N_{N}}{40}\right)^{-\frac{1}{4}}, \quad \text { for } n=4, \\
& v=4.0 \times 10^{-3}\left(\frac{g N_{N}}{40}\right)^{-\frac{1}{10}}, \quad \text { for } n=6, \\
& v=6.7 \times 10^{-3}\left(\frac{g N_{N}}{40}\right)^{-\frac{1}{16}}, \quad \text { for } n=8 .
\end{aligned}
$$

Thus $v$ is larger than $\mu$ for $g<1$ and $n \geq 6$ in contradiction to our assumption that new inflation takes place after hybrid inflation at lower energy scale. For $n=4$ we find that $v$ is smaller than $\mu$ for $g<1$ but it can be close to $\mu$ for $g \lesssim 10^{-3}$. In this case, $\mathcal{R}$ is as large as 0.01 at the onset of new inflation which corresponds to the comoving scale $\ell_{*} \sim 100$ kpc today. As $g$ increases from $10^{-3}, \mathcal{R}$ and $\ell_{*}$ increase. For $g=1$, we find $\mathcal{R} \simeq 0.05$ and $\ell_{*} \simeq 300 \mathrm{kpc}$.

On the other hand, for $c_{N} \neq 0$, we find

$$
\mathcal{R}=\frac{\sqrt{\lambda} \mu^{2}}{2 \pi \sqrt{6} c_{N} v} \cong 1.2 \times 10^{-4} c_{N}^{-1} \frac{\mu}{v},
$$

at the onset of new inflation. It is independent on $n$ and $g$, and is again much larger than $10^{-5}$. The number of $e$-folds depends on these parameters, and for $n=4$ we find

$$
N_{N}=\frac{1}{2 c_{N}} \ln \left(\frac{c_{N} \lambda \mu^{4}}{4 g v^{4}}\right)+\frac{1-4 c_{N}}{2 c_{N}\left(1-c_{N}\right)} .
$$

Thus if we take $c_{N}=0.1$, for example, $N_{N}=40$ implies $v=1.5 \times 10^{-4} g^{-\frac{1}{4}}$, which corresponds to the comoving scale $\ell_{*} \simeq 720 g^{1 / 6} \mathrm{kpc}$ at the onset of new inflation.

\section{DISCUSSION}

In the present paper we have built up a model of double inflation to reproduce a spectrum of adiabatic fluctuations with a running spectral index from $n_{s}>1$ on large scales to $n_{s}<1$ on smaller scales as favored by the recent observational result of the WMAP satellite. Such a spectrum has been known to be realized by the hybrid inflation model in supergravity proposed by Linde and Riotto [7] but we have argued that in their model running of spectral index is too mild to be comparable with the observation. In order to cure this problem we have shown that another inflation is necessary after hybrid inflation and presented a specific model to realize new inflation naturally after hybrid inflation which was originally proposed in [14]. By carefully analyzing the spectrum of fluctuations we have shown that under some natural choice of model parameters the desired spectrum can be realized. In particular, model parameters of hybrid inflation are fully specified from the spectral shape and we find that the energy scale of first inflation is fairly large, $\mu=2.9 \times 10^{-3}$, which means that tensor fluctuations can be detected by PLANCK. On the other hand, it has been pointed out that hybrid inflation has a problem of initial conditions [18]: namely, only a very limited configurations of multi-field space can lead to hybrid inflation unlike chaotic inflation. The resolution of this problem in the present context will be published elsewhere 19]. 
A generic prediction of our double inflation model is that the amplitude of the adiabatic fluctuations is quite large below the scale corresponding to the horizon at the onset of new inflation $\left(<\ell_{*}\right)$. This results in the early formation of dark halo objects with comoving scale $\ell_{*}$. If $\ell_{*}$ is larger than about $1 \mathrm{kpc}$, the dark halos may cause a cosmological problem because they significantly harm subsequent galaxy formation or produce too many gravitational lens events. Our model predicts the critical scale $\ell_{*} \gtrsim 100 \mathrm{kpc}$ which leads to the above cosmological problem. However, the critical scale can be taken much smaller when the uncertainty of the WMAP data is taken into account. As discussed in Sec. II, the WMAP data imply that the uncertainty of the $e$-folds for hybrid inflation is $\Delta N_{H} \sim 8$. Taking a little larger $e$-folds $N_{H} \simeq 15$ gives $\ell_{*}<1 \mathrm{kpc}$, and hence the problem can be avoided. For this case, the predicted small dark halos may play an important role in galaxy formation and early star formation as inferred by WMAP [1]. This subject is beyond the scope of the present paper and will be investigated elsewhere. Another prediction of our model is that contrary to the case of single hybrid inflation, the reheating temperature is low enough to avoid the gravitino problem.

\section{Acknowledgments}

We are grateful to Joe Silk for useful comments. This work was partially supported by the JSPS Grant-in-Aid for Scientific Research No. 14540245 (M.K.), No. 13640285 (J.Y.), and Research Abroad (M.Y.). M.Y. is partially supported by the Department of Energy under Grant No. DEFG0291ER40688.

[1] C. L. Bennett et al., astro-ph/0302207.

[2] D. N. Spergel et al., astro-ph/0302209.

[3] H. V. Peiris et al., astro-ph/0302225

[4] A. H. Guth, Phys. Rev. D 23, 347 (1981); K. Sato, Mon. Not. R. Astron. Soc. 195, 467 (1981).

[5] J. M. Bardeen, Phys. Rev. D 22, 1882 (1980); V. Mukhanov and G. Chibisov, JETP. Lett. 33, 532 (1981).

[6] S. W. Hawking, Phys. Lett. 115B, 295 (1982); A. A. Starobinsky, ibid. 175 (1982); A. H. Guth and S-Y. Pi, Phys. Rev. Lett. 49, 1110 (1982).

[7] A. D. Linde and A. Riotto, Phys. Rev. D 56, 1841 (1997).

[8] B. Feng, M. Li, R. J. Zhang, and X. Zhang, astro-ph/0302479.

[9] B. Kyae and Q. Shafi, astro-ph/0302504

[10] A. D. Linde, Phys. Lett. B 259, 38 (1991); Phys. Rev. D 49, 748 (1994).

[11] A. D. Linde, Phys. Lett. 108B, 389 (1982); A. Albrecht and P. J. Steinhardt, Phys. Rev. Lett. 48, 1220 (1982).

[12] E. J. Copeland, A. R. Liddle, D. H. Lyth, E. D. Stewart, and D. Wands, Phys. Rev. D 49, 6410 (1994).

[13] G. R. Dvali, Q. Shafi, and R. K. Schaefer, Phys. Rev. Lett. 73, 1886 (1994).

[14] K. I. Izawa, M. Kawasaki, and T. Yanagida, Phys. Lett. B 411, 249 (1997).

[15] M. Kawasaki, N. Sugiyama, and T. Yanagida, Phys. Rev. D 57, 6050 (1998); M. Kawasaki and T. Yanagida, ibid. 59, 043512 (1999). 


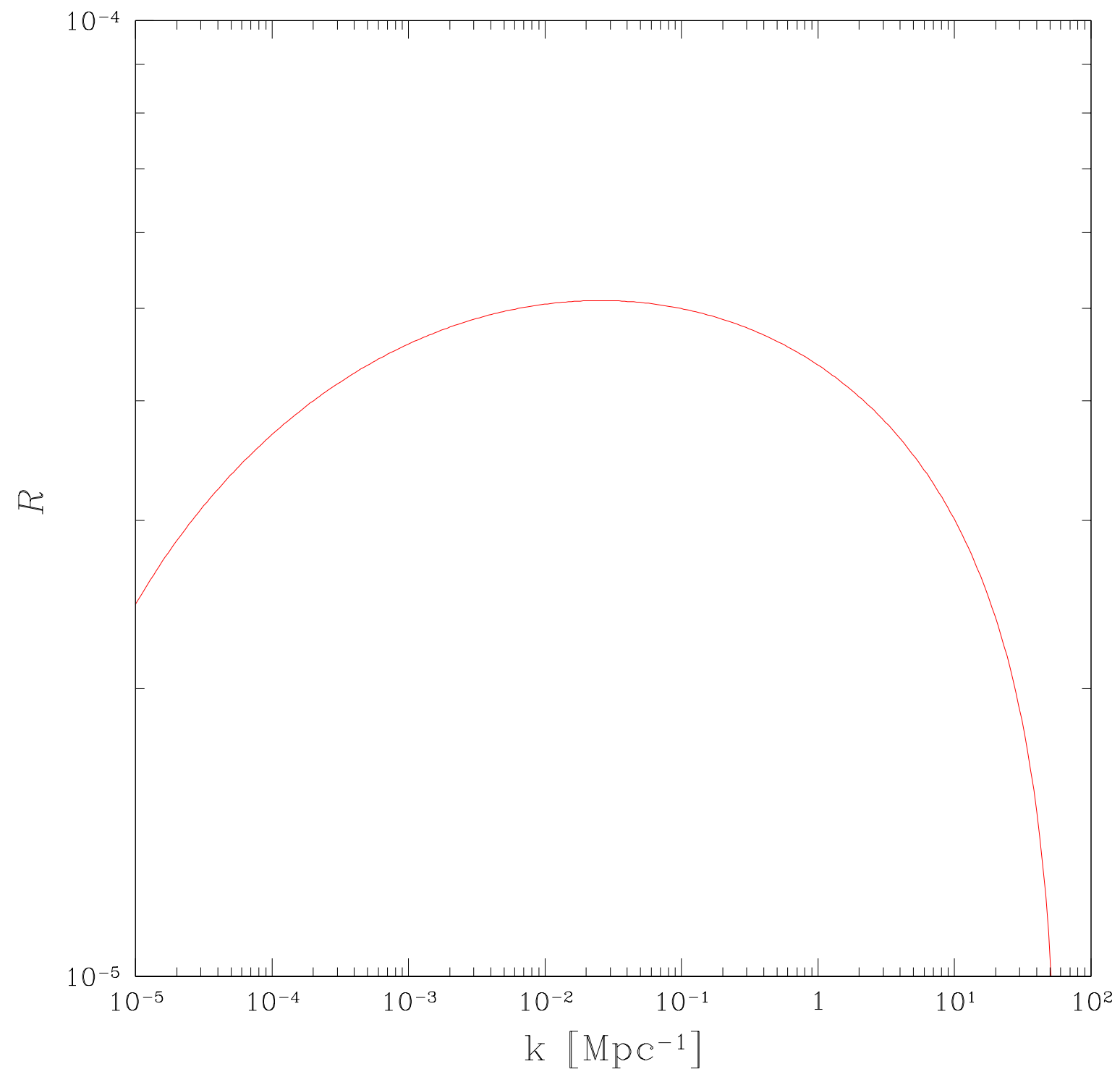

FIG. 1: The amplitude of the curvature fluctuation $\mathcal{R}$ during hybrid inflation is shown with the parameters $\mu=2.9 \times 10^{-3}, \lambda=0.43$. The result is independent of $v$ and $g$ as long as $v \ll \mu$.

[16] T. Kanazawa, M. Kawasaki, N. Sugiyama, and T. Yanagida, Phys. Rev. D 61, 023517 (2000).

[17] T. S. Bunch and P. C. W. Davies, Proc. R. Soc. London A360, 117 (1978).

[18] L. E. Mendes and A. R. Liddle, Phys. Rev. D 62, 103511 (2000); N. Tetradis, ibid. 57, 5997 (1998).

[19] M. Yamaguchi and J. Yokoyama (in preparation). 


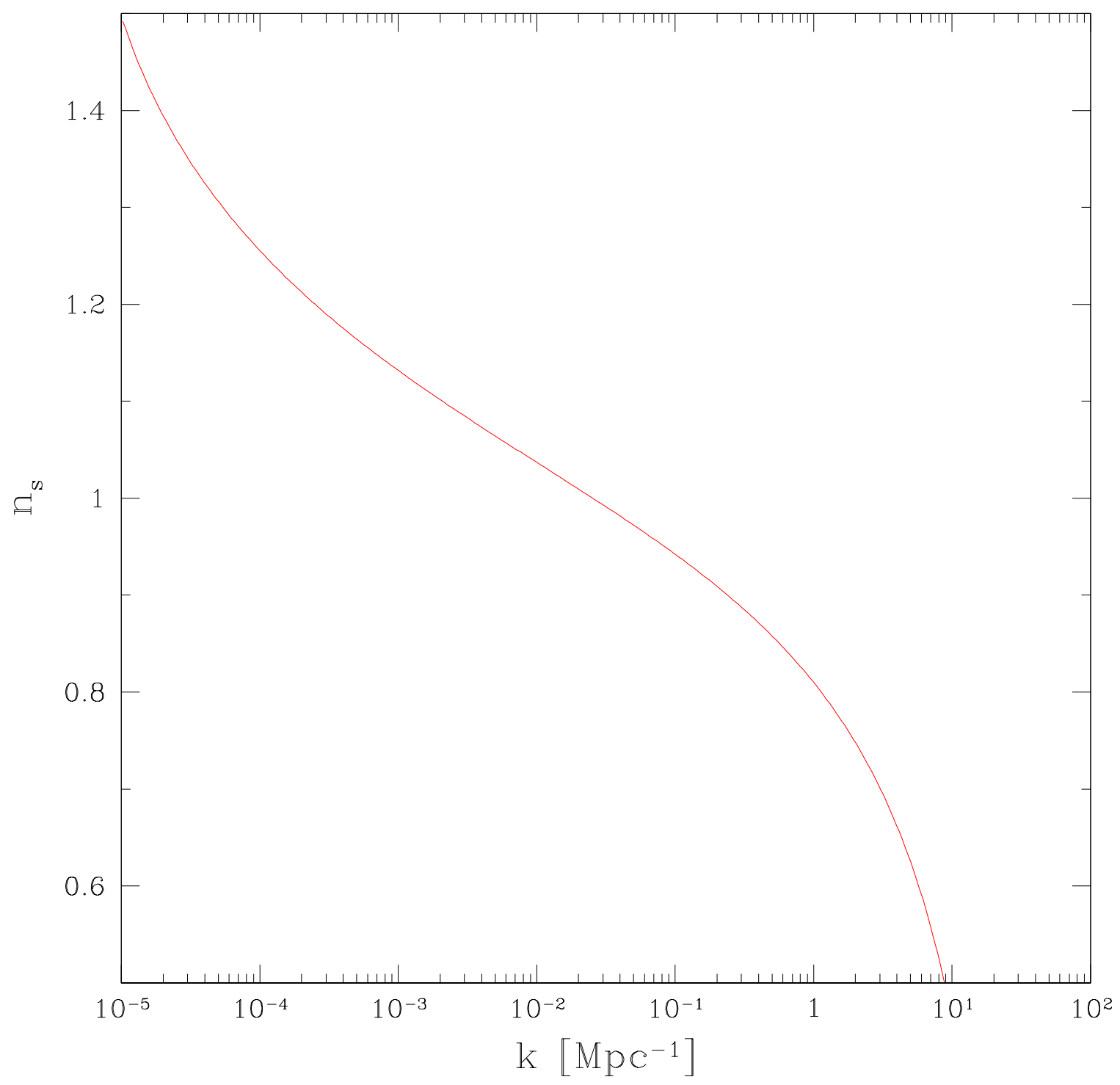

FIG. 2: The spectral index $n_{s}$ during hybrid inflation is shown with the same parameters. 


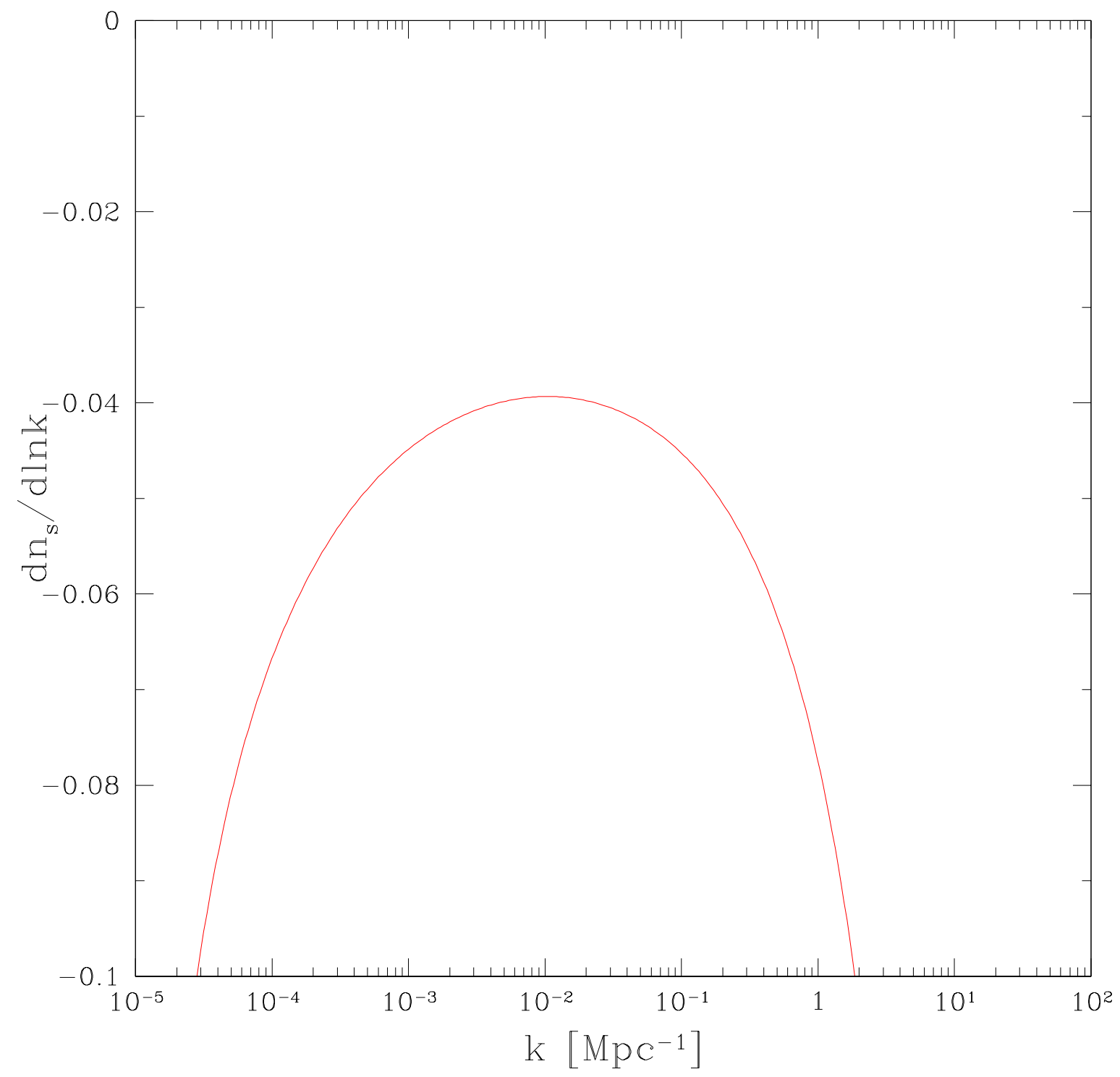

FIG. 3: The derivative of the spectral index $d n_{s} / d \ln k$ during hybrid inflation is shown with the same parameters. 